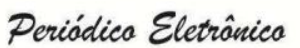

\section{LEVANTAMENTO FLORÍSTICO DE UM REMANESCENTE DE CERRADO EM ÁREA URBANA DE TRÊS LAGOAS-MS, BRASIL.}

\author{
Maria José Neto ${ }^{1}$ \\ Ana Maria Rodrigues Cassiolato ${ }^{2}$ \\ Rodrigo Martins dos Santos ${ }^{3}$
}

\begin{abstract}
RESUMO
O Cerrado é um tipo de vegetação confinado ao Brasil, com apenas poucas extensões que alcançam os vizinhos Paraguai e Bolívia. É um dos tipos de vegetação com maior diversidade no mundo, e nos dias atuais é visto e reconhecido como um "hotspot" para a conservação em escala global. A área urbana de Três Lagoas-MS, Brasil, talvez seja um dos poucos municípios que ainda possui um remanescente de Cerrado típico, no entanto, não figura como área de preservação; é uma área conhecida como Reserva Estratégica do Exército Brasileiro. Com o objetivo de conhecer o potencial da flora deste local, realizou-se o levantamento florístico, que permitiu até o momento, identificar 373 espécies, distribuídas em 231 gêneros e 64 famílias. As famílias mais representadas, com 10 ou mais espécies, foram Fabaceae (58 spp.), Poaceae (24 spp.), Asteraceae (23 spp.), Euphorbiaceae (22 spp.), Myrtaceae (19 spp.), Malvaceae (18 spp.), Bignoniaceae (18 spp.), Apocynaceae (16 spp.), Malpighiaceae (15 spp.) e Rubiaceae (14 spp.). Das espécies identificadas, pelo menos $78 \%$ somam ervas e arbustos, aproximadamente $22 \%$ são árvores ou arvoretas e mais de $75 \%$ apresentam algum valor econômico, parentes silvestres de algumas espécies cultivadas também podem ser vistas na área em questão. Em meio à vegetação típica de Cerrado, encontram-se plantas ruderais e algumas espécies exóticas que em um futuro não muito distante, pode comprometer as plantas desta formação vegetal. Com algum manejo para contensão ou retirada das espécies exóticas, a área deve ser preservada como remanescente de Cerrado típico.
\end{abstract}

PALAVRAS-CHAVE: diversidade florística, cerrado, vegetação urbana.

\section{FLORISTIC SURVEY OF A REMNANT OF CERRADO IN URBAN AREA OF TRÊS LAGOAS / MS - BRAZIL}

\begin{abstract}
The Cerrado is a type of vegetation confined to Brazil, with only a few extensions that reach the Paraguay and Bolivia neighbors. It is one of the types of vegetation with the greatest diversity in the world, and today is seen and recognized as a "hotspot" for the conservation on a global scale. The urban area of Três Lagoas-MS, Brazil, perhaps one of the few cities that still has a remnant of typical Cerrado, however, does not figure as a

\footnotetext{
${ }^{1}$ Prof. ${ }^{\text {a }}$ Drª em Agronomia - Universidade Federal do Mato Grosso do Sul. maria.neto@ufms.br

${ }^{2}$ Prof $^{\mathrm{a}} \mathrm{Dr}^{\mathrm{a}}$ Universidade Estadual Paulista Júlio de Mesquita Filho Campus de llha Solteira anamaria@bio.feis.unesp.br

${ }^{3}$ Graduando em C.Biológicas - Universidade Federal do Mato Grosso do Sul - rodrigoms13@hotmail.com
} 
conservation area; is an area known as the Brazilian Army Strategic Reserve. In order to know the potential of the flora of this place, there was the floristic survey, which allowed so far, identify 373 species belonging to 231 genres and 64 families. The most represented families with 10 or more species were Fabaceae (58 spp.), Poaceae (24 spp.), Asteraceae (23 spp.), Euphorbiaceae (22 spp.), Myrtaceae (19 spp.), Malvaceae (18 spp.), Bignoniaceae (18 spp.), Apocynaceae (16 spp.), Malpighiaceae (15 spp.) and Rubiaceae (14 spp.). Species identified at least $78 \%$ add herbs and shrubs, about $22 \%$ are trees or saplings and over $75 \%$ have some economic value, wild relatives of some cultivated species can also be seen in the area in question. Amid the typical Cerrado vegetation, are ruderal plants and some exotic species that in a not too distant future, may compromise the plants of this plant formation. With some management for containment or removal of exotic species, the area should be preserved as a remnant of typical Cerrado.

KEYWORDS: floristic diversity, cerrado, urban vegetation.

\section{ESTUDIO FLORÍSTICO DE UN REMANENTE DE CERRADO EN ZONA URBANA DE TRÊS LAGOAS / MS - BRASIL}

\section{RESUMEN}

El Cerrado es un tipo de vegetación que se limita a Brasil, con sólo unas pocas extensiones que llegan a los vecinos Paraguay y Bolivia. Es uno de los tipos de vegetación con la mayor diversidad en el mundo, y hoy en día es visto y reconocido como un "hotspot" para la conservación en una escala global. Sostiene especies endémicas y muchos tienen algo de valor económico, como forraje, madera, medicinal u ornamental. El área urbana de Três Lagoas-MS, Brasil, quizás es una de las pocas ciudades que todavía tiene un remanente de típica del Cerrado, pero no figura como área de conservación; es una zona conocida como la Reserva Estratégica del Ejército Brasileño. Con el fin de conocer el potencial de la flora de este lugar, estaba el estudio florístico, que permitía hasta ahora, identificar 373 especies pertenecientes a 231 géneros y 64 familias. Las familias más representadas, con 10 o más especies, fueron Fabaceae (58 spp.), Poaceae (24 spp.), Asteraceae (23 spp.), Euphorbiaceae (22 spp.), Myrtaceae (19 spp.), Malvaceae (18 spp.), Bignoniaceae (18 spp.), Apocynaceae (16 spp.), Malpighiaceae (15 spp.) y Rubiaceae (14 spp.). De las especies identificadas, al menos el $78 \%$ agregan hierbas y arbustos, cerca del 22\% son árboles o árboles jóvenes y más del $75 \%$ tiene algún valor económico; los parientes silvestres de algunas especies cultivadas también se puede ver en la zona en cuestión. En medio de la típica vegetación del Cerrado, hay plantas ruderales y algunas especies exóticas que en un futuro no muy lejano, pueden comprometer las plantas de esta formación vegetal. Con un poco de gestión para la contención o eliminación de especies exóticas, el área debe ser preservada como un remanente del típico Cerrado.

PALABRAS CLAVE: diversidad florística, cerrado, la vegetación urbana

\section{INTRODUÇÃO}

A vegetação do Cerrado, com cerca de 2 milhões de $\mathrm{km}^{2}$ no Brasil, corresponde a $23 \%$ da superfície do país e é excedida apenas pela Floresta Amazônica, que cobre cerca de 3,5 milhões de $\mathrm{km}^{2}$ do território (DURIGAN et al., 2004). É um dos tipos de vegetação com maior diversidade no mundo, estima-se que existam mais de 10.000 espécies de plantas, das quais metade são endêmicas, destas, pelo menos 200 apresentam algum valor econômico, seja forrageiro, madeireiro, medicinal e ornamental (RODRIGUES; RODRIGUES, 2001; HARLEY; GIULIETTI, 2004; SILVA et al., 2001). 


\section{Periódica Eletrânica

Como um "hotspot" para a conservação em escala global, antes de começar a ser destruído, o Cerrado brasileiro tinha 204 milhões de hectares, hoje, 57\% não existe mais (MYERS et al., 2000). O problema é ainda maior porque a taxa de devastação da cobertura vegetal deste bioma continua muito alta. Estima-se que até 2030, o pouco que resta desse domínio de vegetação pode desaparecer sem ao menos ter sido estudada. Além do que, a regeneração natural é comprometida pela presença de gramíneas africanas e fogo. Em apenas quatro décadas, mais da metade da paisagem natural do Cerrado foi modificada (SANO et al., 2001).

\section{OBJETIVOS GERAIS}

Estudar a composição florística de remanescentes de Cerrado, buscando subsídios para conservação deste importante tipo de vegetação.

\section{OBJETIVO ESPECÍFICO}

Atualizar a flora de um remanescente de Cerrado em área urbana, por meio de levantamento florístico.

\section{ÁREA DE ESTUDO}

A área de estudo localiza-se no Município de Três Lagoas-MS. Conhecida como "Reserva do Exército", posiciona-se sob as coordenadas geográficas $20^{\circ} 47^{\prime} 34^{\prime \prime S}$ e $51^{\circ} 41^{\prime} 27^{\prime \prime} O$. Situa-se na área urbana do referido município, precisamente na Avenida Olinto Mancini $n^{\circ} 1317$, com aproximadamente 30 hectares.

A vegetação e os recursos locais são muito importantes para as operações militares, mas merece destaque a preservação desta área pela localização em pleno centro urbano, em Três Lagoas-MS, Brasil.

\section{METODOLOGIA}

Para o levantamento florístico tem sido realizada pelo menos uma excursão a cada semestre, desde 2009 até os dias atuais. Para a descrição da fitofisionomia, utilizou-se o método de observação direta e registros fotográficos. Informações básicas sobre a estrutura da área foram anotadas em cadernetas escolhidas para esta finalidade, e a terminologia usada baseou-se em Ribeiro; Walter (2008). 


\section{Periódica Eletrânica

Durante as excursões procedeu-se observação dos espécimes em fase de reprodução; material estéril também foi fotografado para posteriores observações, quando a identificação no campo não era possível. Durante as coletas foram verificadas e anotadas informações sobre o hábito das plantas, como árvore, arbusto, erva, subarbusto, liana e arvoreta. Utilizou-se o método de caminhamento (FILGUEIRAS et al., 1994) coletar as espécies. Os espécimes coletados foram herborizados de acordo com procedimento convencional e estarão depositados no herbário CEUL da Universidade Federal do Mato Grosso do Sul. Observou-se entre as espécies nativas a presença de algumas plantas ruderais e algumas exóticas.

As plantas foram identificadas por meio de chaves de identificação, bibliografias especializadas e comparação com materiais de herbário. A classificação das espécies em nível de famílias botânicas seguiu o sistema APG III Angiosperm Phylogeny Group III (2009), que posiciona as famílias seguindo suas relações filogenéticas. As grafias dos nomes científicos e seus descritores seguiram os registros das bases de dados do Missouri Botanical Garden e The International Plant Names Index, ambos disponíveis no site The Plant List, acesso ao longo da execução deste trabalho. Os dados obtidos foram organizados em planilhas, a partir das quais foram obtidas tabelas contendo famílias, espécies, porte das plantas e possíveis qualidades agronômicas.

\section{RESULTADOS E DISCUSSÕES}

O presente trabalho representou esforços no sentido de publicar uma listagem atualizada de espécies, da composição da biodiversidade vegetal e de hábitos de crescimento da flora da área estudada. A área da Reserva do Exército apresenta terreno bem drenado, sem presença de corpos d'água; a vegetação pode ser denominada de vegetação de interflúvio (AB'SABER, 1997), e historicamente, não tem sofrido incêndios. O estrato arbóreo é formado por espécies típicas de Cerrado; a maioria dos indivíduos apresenta porte intermediário (com altura média na faixa de 4 - 5 metros) e conformação mais tortuosa. Devido à menor densidade da cobertura arbórea, os seus estratos inferiores mostram-se densos e, geralmente, ricos em espécies arbustivas e herbáceas. Pode ser classificado como Cerrado Sentido Restrito - Cerrado típico. As espécies identificadas para a referida área, com seus 


\section{Periódica Eletrânica

respectivos hábitos e sempre que possível suas qualidades agronômicas estão apresentadas na Tabela 1.

Tabela 1-Relação das famílias, espécies, porte e qualidades agronômicas das plantas em questão.

\begin{tabular}{|c|c|c|}
\hline Família/espécie & Porte & $\begin{array}{l}\text { Qualidade } \\
\text { agronômica }\end{array}$ \\
\hline \multicolumn{3}{|l|}{ Acanthaceae } \\
\hline Ruellia geminiflora Kunth & erva & ornamental \\
\hline Ruellia tweediana Grisebach & erva & ornamental \\
\hline \multicolumn{3}{|l|}{ Amaranthaceae } \\
\hline Froelichia procera (Seub.) T.M. Pedersen & erva & ornamental \\
\hline Gomphrena celosioides Mart. & erva & ornamental \\
\hline Gomphrena macrocephala A.St.-Hil. & subarbustiva & ornamental \\
\hline \multicolumn{3}{|l|}{ Anacardiaceae } \\
\hline Anacardium humile A.St.-Hil. & arbustiva & medicinal \\
\hline Anacardium occidentale L. & arbustiva & frutífera \\
\hline Astronium fraxinifolium Schott & árvore & florestal \\
\hline Myracrodruon urundeuva Allemão & árvore & florestal \\
\hline \multicolumn{3}{|l|}{ Annonaceae } \\
\hline Annona coriacea Mart. & árvore & frutífera \\
\hline Annona crassiflora Mart. & árvore & florestal, med. e alim. \\
\hline Annona dioica A.St.-Hil. & subarbusto & medicinal \\
\hline Annona nutans (R.E.Fr.) R.E.Fr. & subarbusto & \\
\hline Annona tomentosa R.E.Fr. & subarbusto & \\
\hline Annona warmingiana Mello-Silva \& Pirani & subarbusto & \\
\hline Duguetia furfuracea (A.St.-Hil.) Saff. & arbusto & frutífera e medicinal \\
\hline Duguetia lanceolata A.St.-Hil. & arbusto & frutífera \\
\hline Xylopia aromatica (Lam.) Mart. & árvore & medicinal \\
\hline \multicolumn{3}{|l|}{ Apocynaceae } \\
\hline Allagoptera campestris (Mart.) Kuntze & palm/folhas basais & ornamental \\
\hline Aspidosperma macrocarpon Mart. & árvore & florestal \\
\hline Aspidosperma tomentosum Mart. & árvore & florestal \\
\hline Blepharodon bicuspidatum E. Fourn. & trepadeira & \\
\hline Blepharodon nitidum (Vell.) J.F.Macbr. & trepadeira & \\
\hline \multicolumn{3}{|l|}{ Forsteronia pubescens D.C. } \\
\hline Hancornia speciosa Gomes & árvore & frutífera \\
\hline Himatanthus obovatus (Müll.Arg.) Woodson & arvoreta & ornamental \\
\hline Mandevilla coccinea (Hook \& Arn.) Woodson & erva/xilopódio & ornamental \\
\hline Mandevilla illustris (Vell.) Woodson & erva/xilopódio & ornamental \\
\hline Mandevilla longiflora (Desf.) Pichon & erva/xilopódio & orn. e medicinal \\
\hline Odontadenia lutea (Vell.) Marckgr. & trepadeira & ornamental \\
\hline
\end{tabular}




\section{Periódica Eletrônica

Continuação...Tabela 1

\begin{tabular}{|c|c|c|}
\hline Família/espécie & Porte & $\begin{array}{l}\text { Qualidade } \\
\text { agronômica }\end{array}$ \\
\hline Odontadenia nitida (Vell.) Müll.Arg. & trepadeira & ornamental \\
\hline Oxipetalum sp. & trepadeira & alimentícia \\
\hline Prestonia erecta J.F.Morales & subarbusto/xilopódio & ornamental \\
\hline Prestonia tomentosa R.Br. & trepadeira & ornamental \\
\hline $\begin{array}{l}\text { Secondatia densiflora A.DC. } \\
\text { Arecaceae }\end{array}$ & trepadeira & ornamental \\
\hline Acrocomia aculeata (Jacq.) Lodd. ex Mart. & palmeira & alimentícia \\
\hline $\begin{array}{l}\text { Cocos nucifera L. } \\
\text { Aristolochiaceae }\end{array}$ & palmeira & alimentícia \\
\hline Aristolochia esperanzae Kuntze & trepadeira & medicinal \\
\hline Aristolochia sp. & trepadeira & ornamental \\
\hline \multicolumn{3}{|l|}{ Asteraceae } \\
\hline Acanthospermum australe (Loefl.) Kuntze & erva & medicinal \\
\hline Aspilia foliacea (Spreng.) Baker & erva & ornamental \\
\hline Bidens gardneri Baker & erva & orn. e medicinal \\
\hline Bidens subalternans DC. & erva & medicinal \\
\hline Calyptocarpus biaristatus (DC.) H.Rob. & subarbusto & \\
\hline Eclipta prostrata (L.) L. & erva & \\
\hline Elephantopus mollis Kunth & herbácea & medicinal \\
\hline Emilia fosbergii Nicolson & erva & medicinal \\
\hline Erechtites hieraciifolius (L.) Raf. ex DC. & erva & \\
\hline $\begin{array}{l}\text { Chromolaena maximilianii (Schrad. ex DC.) R.M.King } \\
\text { \& H.Rob. }\end{array}$ & arbusto & medicinal \\
\hline Eupatorium pauciflorum (Raf.) Raf. & erva & \\
\hline Eupatorium squalidum (Raf.) Raf. & subarbusto & \\
\hline Gochnatia barrosii Cabrera & arbusto grande & medicinal \\
\hline Gochnatia polymorpha (Less.) Cabrera & arvoreta & \\
\hline Gochnatia pulchra Cabrera & arbusto & \\
\hline Parthenium hysterophorus L. & erva & \\
\hline Piptocarpha rotundifolia (Less.) Baker & arbusto & \\
\hline Porophyllum ruderale (Jacq.) Cass. & erva & medicinal \\
\hline Pterocaulon virgatum (L.) DC. & erva & ornamental \\
\hline \multicolumn{3}{|l|}{ Synedrellopsis grisebachii Hieron. \& Kuntze ex } \\
\hline O.Hoffm. & erva & \\
\hline Tridax procumbens (L.) L. & erva & \\
\hline Vernonia scabra K.Schum. & arbusto & medicinal \\
\hline \multicolumn{2}{|l|}{ Bignoniaceae } & ornamental \\
\hline Amphilophium elongatum (Vahl) L.G.Lohmann & trepadeira & ornamental \\
\hline Amphilophium paniculatum (L.) Kunth & trepadeira & ornamental \\
\hline Anemopaegma arvense (Vell.) Stellfeld ex de Souza. & subarbusto & medicinal \\
\hline Anemopaegma glaucum Mart. Ex DC. & subarbusto & medicinal \\
\hline Cybistax antisyphilitica (Mart.) Mart. & árvore & florestal \\
\hline Dolichandra quadrivalvis (Jacq.) L.G.Lohmann & trepadeira & ornamental \\
\hline Fridericia florida (DC.) L. G. Lohmann & trepadeira & ornamental \\
\hline Fridericia platyphylla (Cham.) L.G.Lohmann & arbusto & ornamental \\
\hline Jacaranda caroba (Vell.) A.DC. & arbusto & medicinal \\
\hline Jacaranda cuspidifolia Mart. & árvore & ornamental \\
\hline Jacaranda decurrens Cham. & subarbusto & orn. e medicinal \\
\hline Jacaranda rufa Silva Manso & subarbusto & ornamental \\
\hline
\end{tabular}




\begin{tabular}{|c|c|c|}
\hline Família/espécie & Porte & $\begin{array}{l}\text { Qualidade } \\
\text { agronômica }\end{array}$ \\
\hline Pyrostegia venusta (Ker Gawl.) Miers & trepadeira & orn. e medicinal \\
\hline Tabebuia aurea (Silva Manso) Benth. \& Hook.f. ex S.Moore & árvore & medicinal e florestal \\
\hline Tabebuia ochracea A.H. Gentry & árvore & medicinal e florestal \\
\hline Tanaecium pyramidatum (Rich.) L.G.Lohmann & trepadeira & \\
\hline Zeyheria montana Mart. & arbusto & ornamental \\
\hline Bixaceae & & \\
\hline $\begin{array}{l}\text { Cochlospermum regium (Schrank) Pilg. } \\
\text { Boraginaceae }\end{array}$ & arbusto & med. e ornamental \\
\hline Heliotropium procumbens Mill. & erva & \\
\hline Brassicaceae & & \\
\hline $\begin{array}{l}\text { Cleome affinis DC } \\
\text { Bromeliaceae }\end{array}$ & erva & \\
\hline Ananas ananassoides (Baker) L.B.Sm. & erva rosulada & ornamental \\
\hline Bromelia balansae Mez & erva rosulada & ornamental \\
\hline Dyckia tuberosa (Vell.) Beer & erva rosulada & ornamental \\
\hline Tillandsia duratii Vis. & epífita & ornamental \\
\hline Tillandsia usneoides (L.) L. & epífita & ornamental \\
\hline Burseraceae & & \\
\hline $\begin{array}{l}\text { Protium ovatum Engl. } \\
\text { Cactaceae }\end{array}$ & arbusto & medicinal \\
\hline $\begin{array}{l}\text { Cereus hildmannianus K.Schum. } \\
\text { Praecereus euchlorus (F.A.C.Weber ex K.Schum.) }\end{array}$ & ereta & orn. e alimentícia \\
\hline $\begin{array}{l}\text { N.P.Taylor } \\
\text { Caryocaraceae }\end{array}$ & ereta & orn. e alimentícia \\
\hline $\begin{array}{l}\text { Caryocar brasiliense A.St.-Hil. } \\
\text { Caryophyllaceae }\end{array}$ & árvore & florestal, alim. e med \\
\hline $\begin{array}{l}\text { Polycarpaea corymbosa (L.) Lam. } \\
\text { Celastraceae }\end{array}$ & erva & ornamental \\
\hline Austroplenckia populnea (Reissek) Lundell & árvore & medicinal \\
\hline Peritassa campestris (Cambess.) A.C.Sm. & arbusto & \\
\hline Salacia crassifolia (Mart. ex Schult.) G. Don & arbusto & \\
\hline Tontelea micrantha (Mart. ex Schult.) A.C. Sm. & arbusto & \\
\hline Chrysobalanaceae & & \\
\hline Couepia grandiflora (Mart. \& Zucc.) Benth. \& Hook.f. & árvore & florestal \\
\hline Licania humilis Cham. \& Schltdl. & árvore & florestal \\
\hline Parinari obtusifolia Hook.f. & subarbusto & \\
\hline Clusiaceae & & \\
\hline Kielmeyera coriacea Mart. & árvore & florestal, orn. e med. \\
\hline $\begin{array}{l}\text { Kielmeyera grandiflora (Wawra) Saddi } \\
\text { Combretaceae }\end{array}$ & árvore & florestal, orn. e med. \\
\hline Buchenavia tomentosa Eichler & árvore & florestal \\
\hline Terminalia argentea Mart. & árvore & florestal \\
\hline Terminalia glabrescens Mart. & árvore & florestal \\
\hline Commelinaceae & & \\
\hline Commelina benghalensis L. & erva & \\
\hline Commelina erecta $\mathrm{L}$. & erva & med. e ornamental \\
\hline $\begin{array}{l}\text { Murdannia nudiflora (L.) Brenan } \\
\text { Connaraceae }\end{array}$ & erva & medicinal \\
\hline Connarus suberosus Planch. & arbusto & florestal \\
\hline Rourea induta Planch. & arbusto & medicinal \\
\hline
\end{tabular}




\section{Periódica Eletrânica

Continuação...Tabela 1

\section{Família/espécie}

Ipomoea procumbens Mart.ex Choisy

Jacquemontia tamnifolia (L.) Griseb.

Merremia cissoides (Lam.) Hallier f.

\section{Cucurbitaceae}

Momordica charantia L.

Melancium campestre Naudin

Cyperaceae

Bulbostylis paradoxa (Spreng.) Lindm.

Bulbostylis capillaris (L.) Kunth ex C.B.Clarke

Cyperus difformis $\mathrm{L}$.

Cyperus meyenianus Kunth

Cyperus laxus Lam.

Fuirena umbellata Rottb.

Kyllinga odorata Vahl

Rhynchospora corymbosa (L.) Britton

Rhynchospora nervosa (Vahl) Boeckeler

\section{Dilleniaceae}

Curatella americana $\mathrm{L}$.

Davilla elliptica A.St.-Hil.

\section{Ebenaceae}

Diospyros brasiliensis Mart.ex Miq.

Diospyros hispida A.DC.

Erythroxylaceae

Erythroxylum campestre A.St.-Hil.

Erythroxylum suberosum A.St.-Hil.

Erythroxylum tortuosum Mart.

Euphorbiaceae

Astraea lobata (L.) Klotzsch

Cnidoscolus albomaculatus (Pax) I.M.Johnst.

Cnidoscolus appendiculatus (Pax \& K.Hoffm.) Pax \&

K.Hoffm.

Croton antisyphiliticus Mart.

Croton bonplandianus Baill.

Croton campestris A.St.-Hil.

Croton glandulosus $\mathrm{L}$.

Croton goyazensis Müll.Arg.

Euphorbia heterophylla L.

Euphorbia hirta L.

Euphorbia hyssopifolia L.

Euphorbia prostrata Aiton

Jatropha elliptica (Pohl) Oken

Mabea fistulifera Mart.

Manihot caerulescens Pohl.

Manihot gracilis Pohl.

Manihot tripartita (Spreng.) Müll.Arg.

Maprounea guianensis Aubl.

Microstachys corniculata (Vahl) Griseb.

Ricinus communis L.

Sapium haematospermum Müll.Arg.

Albizia lebbeck (L.) Benth.

\section{Porte}

erva

erva

erva

erva

erva

erva

erva

erva

erva

erva

erva

erva

erva

erva

árvore

arbusto

arvoreta

arvoreta

arbusto

arbusto/arvoreta

arbusto/arvoreta

erva

subarbusto

subarbusto

subarbusto

erva

arbusto

erva

subarbusto

erva

erva

erva

erva

subarbusto

árvore

arbusto

subarbusto

arbusto

árvore

erva

arbusto

árvore

árvore

\section{Qualidade} agronômica

ornamental

ornamental

ornamental

florestal

med. e ornamental

alimentícia

alimentícia

medicinal

medicinal

medicinal

medicinal

medicinal

medicinal

florestal

recurso genético

recurso genético

recurso genético

florestal

exótica 


\section{Periódica Eletrânica

Continuação...Tabela 1

\begin{tabular}{|c|c|c|}
\hline Família/espécie & Porte & $\begin{array}{l}\text { Qualidade } \\
\text { agronômica }\end{array}$ \\
\hline Anadenanthera falcata (Benth.) Speg. & árvore & florestal \\
\hline Bauhinia pentandra (Bong.) Steud. & arbusto & medicinal \\
\hline Bauhinia rufa (Bong.) Steud. & arbusto & medicinal \\
\hline Chamaecrista campestris H.S.Irwin \& Barneby & erva & ornamental \\
\hline Chamaecrista desvauxii (Collad.) Killip & subarbusto & forrageira \\
\hline Chamaecrista flexuosa (L.) Greene & subarbusto & forrageira \\
\hline Chamaecrista nictitans (L.) Moench & erva & forrageira \\
\hline Chamaecrista rotundifolia (Pers.) Greene & erva & medicinal \\
\hline Chamaecrista serpens (L.) Greene & erva & forrageira \\
\hline Copaifera langsdorffii Desf. & árvore & florestal e med. \\
\hline Copaifera martii Hayne & arvoreta & medicinal \\
\hline Crotalaria incana $\mathrm{L}$. & subarbusto & forrageira \\
\hline Crotalaria longifolia Lam. & subarbusto & forrageira \\
\hline Crotalaria micans Link & subarbusto & forrageira \\
\hline Crotalaria pallida Aiton & subarbusto & forrageira \\
\hline Crotalaria stipularia Desv. & erva & forrageira \\
\hline Desmodium adscendens (Sw.) DC. & subarbusto & medicinal \\
\hline Desmodium incanum DC. & erva & medicinal \\
\hline Desmodium tortuosum (Sw.) DC. & subarbusto & medicinal \\
\hline Dimorphandra mollis Benth & árvore & florestal e ornamental \\
\hline Diptychandra aurantiaca Tul. & árvore & florestal \\
\hline Enterolobium contortisiliquum (Vell.) Morong & árvore & florestal \\
\hline Enterolobium gummiferum (Mart.) J.F.Macbr. & árvore & florestal \\
\hline Eriosema crinitum (Kunth) G.Don & erva & forrageira \\
\hline Galactia eriosematoides Harms. & subarbusto & medicinal \\
\hline Hymenaea stigonocarpa Hayne & árvore & florestal, alim. e med. \\
\hline Indigofera hirsuta L. & subarbusto & forrageira \\
\hline Indigofera suffruticosa Mill. & subarbusto & forrageira e medicinal \\
\hline Inga laurina (Sw.) Willd. & árvore & \\
\hline Machaerium acutifolium Vogel & árvore & florestal \\
\hline Macroptilium atropurpureum Urb. Urb. & erva & forrageira \\
\hline Mimosa adenocarpa Benth. & arbusto & ornamental \\
\hline Mimosa debilis Willd. & subarbusto & ornamental \\
\hline Mimosa hebecarpa Benth. & arvoreta & ornamental e florestal \\
\hline Mimosa nuda Benth. & arbusto & ornamental \\
\hline Mimosa polycarpa Kunth & arbusto & ornamental \\
\hline Mimosa quadrivalvis L. & erva & ornamental \\
\hline Mimosa xanthocentra Mart. & subarbusto & ornamental \\
\hline Plathymenia reticulata Benth. & árvore & florestal \\
\hline Senna occidentalis (L.) Link & arbusto & medicinal \\
\hline Senna rugosa (G. Don) H.S.Irwin \& Barneby & arbusto & medicinal \\
\hline Senna silvestris (Vell.) H.S.Irwin \& Barneby & arbusto & ornamental \\
\hline Senna velutina (Vogel) H.S.Irwin \& Barneby & arbusto & ornamental \\
\hline Stryphnodendron adstringens (Mart.) Coville & árvore & florestal e medicinal \\
\hline Stryphnodendron obovatum Benth. & árvore & florestal e medicinal \\
\hline Stryphnodendron polyphyllum Mart. & árvore & florestal e medicinal \\
\hline Stylosanthes acuminata M.B.Ferreira \& Sousa Costa & subarbusto & forrageira \\
\hline Stylosanthes guianensis (Aubl.) Sw. & subarbusto & forrageira \\
\hline Stylosanthes leiocarpa Vogel & erva & forrageira \\
\hline Stylosanthes viscosa Sw. & subarbusto & forrageira \\
\hline
\end{tabular}




\section{Periódica Eletrânica

Continuação...Tabela 1

\begin{tabular}{|c|c|c|}
\hline Família/espécie & Porte & $\begin{array}{l}\text { Qualidade } \\
\text { agronômica }\end{array}$ \\
\hline Teramnus volubilis Sw. & erva & forrageira \\
\hline Vatairea macrocarpa (Benth.) Ducke & árvore & florestal \\
\hline Vigna peduncularis (Kunth) Fawc. \& Rendle & erva & forrageira \\
\hline Zornia crinita (Mohlenbr.) Vanni & erva & forrageira \\
\hline Zornia glochidiata DC. & erva & \\
\hline Zornia latifolia Sm. & erva & forrageira \\
\hline Zornia reticulata $\mathrm{Sm}$. & erva & forrageira \\
\hline \multicolumn{3}{|l|}{ Iridaceae } \\
\hline Cipura paludosa Aubl. & erva & ornamental \\
\hline \multicolumn{3}{|l|}{ Lamiaceae } \\
\hline Aegiphila Ihotzkiana Cham. & arvoreta & ornamental \\
\hline Amasonia campestris (Aubl.) Moldenke & subarbusto & ornamental \\
\hline Hyptis mutabilis (Rich.) Briq. & subarbusto & medicinal \\
\hline Hyptis suaveolens (L.) Poit. & subarbusto & medicinal \\
\hline Hyptis villosa Pohl ex Benth. & subarbusto & medicinal \\
\hline Peltodon tomentosus Pohl & subarbusto & medicinal \\
\hline \multicolumn{3}{|l|}{ Lecythidaceae } \\
\hline $\begin{array}{l}\text { Eschweilera nana (O.Berg) Miers } \\
\text { Loganiaceae }\end{array}$ & arvoreta & orn. e alimentícia \\
\hline Strychnos brasiliensis (Spreng.) Mart. & arbusto & medicinal \\
\hline Strychnos pseudoquina A.St.-Hil. & arb até árvore & medicinal \\
\hline \multicolumn{3}{|l|}{ Lythraceae } \\
\hline Cuphea carthagenensis (Jacq) J.F.Macbr. & erva & medicinal \\
\hline Lafoensia pacari A.St.-Hil. & árvore & florestal e medicinal \\
\hline \multicolumn{3}{|l|}{ Malpighiaceae } \\
\hline Banisteriopsis campestris (A.Juss.) Little & arbusto & ornamental \\
\hline Byrsonima coccolobifolia Kunth & árvore & flor. e ornamental \\
\hline Byrsonima crassifolia (L.) Kunth & arbusto & ornamental \\
\hline Byrsonima guilleminiana A.Juss. & arbusto & ornamental \\
\hline Byrsonima intermedia A.Juss. & arbusto & medicinal \\
\hline Byrsonima subterranea Brade \& Markgr. & subarbusto & ornamental \\
\hline Byrsonima verbascifolia (L.) DC. & arvoreta & medicinal \\
\hline Camarea affinis A.St.-Hil. & erva com xilopódio & ornamental \\
\hline \multicolumn{3}{|l|}{ Diplopterys pubipetala (A.Juss.) W.R.Anderson \& } \\
\hline C.Davis & arbusto & ornamental \\
\hline Galphimia australis Chodat & erva com xilopódio & ornamental \\
\hline Heteropterys aphrodisiaca Machado & arbusto & medicinal \\
\hline Heteropterys byrsonimifolia A.Juss. & arbusto & ornamental \\
\hline Peixotoa cordistipula A.Juss. & arbusto & \\
\hline Peixotoa reticulata Griseb. & liana & ornamental \\
\hline Tetrapterys salicifolia Nied. & erva com xilopódio & \\
\hline \multicolumn{3}{|l|}{ Malvaceae } \\
\hline Abutilon bedfordianum (Hook.) A.St.-Hil. & subarbusto & ornamental \\
\hline Ayenia tomentosa L. & erva & \\
\hline Corchorus orinocensis Kunth & erva & ornamental \\
\hline Eriotheca gracilipes (K.Schum.) A.Robyns & árvore & florestal \\
\hline Malvastrum coromandelianum (L.) Garcke & erva a subarbusto & \\
\hline Melochia parvifolia Kunth & arbusto & ornamental e fibras \\
\hline $\begin{array}{l}\text { Melochia pilosa (Mill.) Fawe \& Rendl. } \\
\text { Melochia spicata (L.) Fryxell }\end{array}$ & subarbusto & ornamental \\
\hline
\end{tabular}


Continuação...Tabela 1

\begin{tabular}{|c|c|c|}
\hline Família/espécie & Porte & $\begin{array}{l}\text { Qualidade } \\
\text { agronômica }\end{array}$ \\
\hline Pavonia cancellata (L.) Cav. & erva & ornamental \\
\hline Pavonia guerkeana R.E.Fr. & subarbusto & ornamental \\
\hline Pavonia sidifolia Kunth & erva & ornamental \\
\hline Sida cerradoensis Krap. & erva & \\
\hline Sida glaziovii K. Schum & erva & \\
\hline Sida linifolia Juss. ex Cav. & erva & \\
\hline Sidastrum paniculatum (L.) Fryxell & subarbusto & \\
\hline Urena lobata L & subarbusto & medicinal \\
\hline Waltheria communis A.St.-Hil. & subarbusto & medicinal \\
\hline Waltheria indica $\mathrm{L}$. & subarbusto & medicinal \\
\hline \multicolumn{3}{|l|}{ Melastomataceae } \\
\hline Miconia albicans (Sw.) Steud. & arvoreta & ornamental \\
\hline Mouriri elliptica Mart. & arvoreta & alimentícia \\
\hline \multicolumn{3}{|l|}{ Menispermaceae } \\
\hline Cissampelos ovalifolia DC. & subarbusto & medicinal \\
\hline \multicolumn{3}{|l|}{ Molluainaceae } \\
\hline Mollugo verticillata $\mathrm{L}$ & erva & \\
\hline \multicolumn{3}{|l|}{ Moraceae } \\
\hline $\begin{array}{l}\text { Brosimum gaudichaudii Trécul } \\
\text { Myrtaceae }\end{array}$ & arbusto & alim. e medicinal \\
\hline Campomanesia adamantium (Cambess.) O. Berg & arbusto & alimentícia \\
\hline Campomanesia sessiliflora (O.Berg) Mattos & arbusto & alimentícia \\
\hline Corymbia citriodora (Hook.) K.D.Hill \& L.A.S.Johnson & árvore & florestal/exótica \\
\hline Eugenia aurata O.Berg. & arvoreta & alimentícia \\
\hline Eugenia bimarginata DC. & arbusto & alimentícia \\
\hline Eugenia calycina Cambess. & subarbusto & alimentícia \\
\hline Eugenia dysenterica DC & arbusto & alimentícia \\
\hline Eugenia egensis DC. & arbusto & alimentícia \\
\hline Eugenia lutescens Cambess. & subarbusto & alimentícia \\
\hline Eugenia pitanga (O.Berg) Nied. & arbusto & alimentícia \\
\hline Eugenia punicifolia (Kunth) DC. & arbusto & alim. e medicinal \\
\hline Eugenia stictopetala DC. & arbusto & alimentícia \\
\hline Myrcia bella Cambess. & arvoreta & alimentícia \\
\hline Myrcia linearifolia Cambess. & arbusto peq. & orn. e alimentícia \\
\hline Myrciaria cuspidata O.Berg. & arbusto & alimentícia \\
\hline Psidium grandifolium Mart. ex DC. & arbusto peq. & alimentícia \\
\hline Psidium guajava L. & árvore & alimentícia \\
\hline Psidium guineense Sw. & arbusto & alimentícia \\
\hline Syzygium aqueum (Burm.f.) Alston & arbusto & alimentícia \\
\hline \multicolumn{3}{|l|}{ Nictaginaceae } \\
\hline Boerhavia diffusa L. & erva & \\
\hline Guapira noxia (Netto) Lundell & arvoreta & florestal \\
\hline Neea theifera Oerst. & arvoreta & florestal \\
\hline \multicolumn{3}{|l|}{ Ochnaceae } \\
\hline Ouratea spectabilis (Mart. ex Engl.) Engl. & árvore & flor. e ornamental \\
\hline Ouratea floribunda Engl. & arbusto & ornamental \\
\hline Ouratea hexasperma (A.St. Hil.) Baill. & árvore & flor. e ornamental \\
\hline Opiliaceae & & \\
\hline $\begin{array}{l}\text { Agonandra brasiliensis Miers ex Benth. } \\
\text { Orchidaceae }\end{array}$ & árvore & florestal \\
\hline
\end{tabular}




\section{Periódica Eletranica

Continuação...Tabela 1

\begin{tabular}{|c|c|c|}
\hline Família/espécie & Porte & $\begin{array}{l}\text { Qualidade } \\
\text { agronômica }\end{array}$ \\
\hline $\begin{array}{l}\text { Catasetum barbatum (Lindl.) Lindl. } \\
\text { Passifloraceae }\end{array}$ & epífita & ornamental \\
\hline Passiflora cincinnata Mast. & trepadeira & alimentícia \\
\hline Passiflora foetida L. & trepadeira & recurso genético \\
\hline Passiflora pohlii Mast. & trepadeira & recurso genético \\
\hline Piriqueta rosea (A. St.-Hil., A. Juss. \& Cambess.) Urb. & erva & ornamental \\
\hline Piriqueta cistoides (L.) Griseb. & erva & ornamental \\
\hline $\begin{array}{l}\text { Turnera melochioides A. St.-Hil. \& Cambess. } \\
\text { Phyllanthaceae }\end{array}$ & erva & ornamental \\
\hline $\begin{array}{l}\text { Phyllanthus orbiculatus L.C. Rich. } \\
\text { Plantaginaceae }\end{array}$ & erva & medicinal \\
\hline $\begin{array}{l}\text { Scoparia dulcis L. } \\
\text { Poaceae }\end{array}$ & erva & \\
\hline Andropogon bicornis L. & erva & forrageiro \\
\hline Axonopus pressus (Steud.) Parodi & erva & forrageiro \\
\hline Brachiaria brizantha (A.Rich.) Stapf & erva & exótica \\
\hline Brachiaria decumbens Stapf & erva & exótica \\
\hline Cenchrus echinatus L. & erva & \\
\hline Chloris barbata (L.) Sw. & erva & forrageiro \\
\hline Cynodon dactylon (L.) Pers. & erva & forrageiro \\
\hline Dactyloctenium aegyptium (L.) Willd. & erva & forrageiro \\
\hline Digitaria ciliaris (Retz.) Koel. & erva & forrageiro \\
\hline Digitaria insularis (L.) Mez ex Ekman & erva & forrageiro \\
\hline Echinolaena inflexa (Poir.) Chase. & erva & forrageiro \\
\hline Eleusine indica (L.) Gaertn. & erva & forrageiro \\
\hline Eragrostis pilosa (L.) P.Beauv. & erva & forrageiro \\
\hline Eustachys distichophylla (Lag.) Nees & erva & forrageiro \\
\hline Gymnopogon foliosus (Willd.) Nees & erva & forrageiro \\
\hline Hyparrhenia rufa (Nees) Stapf & erva & forrageiro \\
\hline Loudetiopsis chrysothrix (Nees) Conert & erva & forrageiro \\
\hline Melinis minutiflora P. Beauv. & erva & exótica \\
\hline Melinis repens (Willd.) Zizka & erva & forrageiro \\
\hline Panicum maximum Jacq. & erva & forrageiro \\
\hline Panicum sellowii Nees & erva & forrageiro \\
\hline Paspalum notatum Flüggé & erva & forrageiro \\
\hline Pennisetum polystachion (L.) Schult. & erva & forrageiro \\
\hline Sporobolus indicus (L.) R. Br. & erva & forrageiro \\
\hline Polygalaceae & & \\
\hline Bredemeyera floribunda Willd. & arbusto & medicinal \\
\hline Monnina richardiana A. St.-Hil. & subarbusto & \\
\hline Polygala violacea Aubl. & erva & medicinal \\
\hline Polygonaceae & & \\
\hline Coccoloba mollis Casar. & árvore & florestal \\
\hline Portulacaceae & & \\
\hline Portulaca fluvialis D. Legrand & erva & ornamental \\
\hline Portulaca oleracea L. & erva & \\
\hline Proteaceae & & \\
\hline $\begin{array}{l}\text { Roupala montana Aubl. } \\
\text { Rubiaceae }\end{array}$ & árvore & florestal \\
\hline Alibertia edulis (Rich.) A.Rich. ex DC. & arbusto & alimentícia \\
\hline
\end{tabular}




\section{Periódica Eletrânica

Continuação...Tabela 1

\begin{tabular}{|c|c|c|}
\hline Família/espécie & Porte & $\begin{array}{l}\text { Qualidade } \\
\text { agronômica }\end{array}$ \\
\hline Cordiera elliptica (Cham.) Kuntze & subarbusto & alimentícia \\
\hline Cordiera sessilis (Vell.) Kuntze & arbusto & medicinal \\
\hline Declieuxia fruticosa (Willd. ex Roem. \& Schult.) Kuntze & subarbusto & ornamental \\
\hline Diodella teres (Walter) Small & erva & \\
\hline Guettarda viburnoides Cham. \& Schltdl. & arvoreta & \\
\hline Palicourea rigida Kunth & subarbusto & ornamental \\
\hline Richardia brasilensis Gomes & erva & \\
\hline Richardia grandiflora (Cham. \& Schltdl.) Steud. & erva & \\
\hline Spermacoce alata Aubl. & erva & \\
\hline Spermacoce capitata Ruiz \& Pav. & erva a subarbusto & ornamental \\
\hline Staelia thymoides Cham. \& Schltdl. & erva & \\
\hline Tocoyena brasiliensis Mart. & arbusto & ornamental \\
\hline Tocoyena formosa (Cham. \& Schltdl.) K.Schum. & arbusto & ornamental \\
\hline \multicolumn{3}{|l|}{ Rutaceae } \\
\hline Citrus limon (L.) Osbeck & arvoreta & alim. e medicinal/exótica \\
\hline \multicolumn{3}{|l|}{ Salicaceae } \\
\hline Casearia sylvestris Sw. & arvoreta & medicinal \\
\hline \multicolumn{3}{|l|}{ Sapindaceae } \\
\hline Allophylus edulis (A.St.-Hil., A.Juss. \& Cambess.) Radlk. & arvoreta & florestal \\
\hline Magonia pubescens A.St.-Hil. & árvore & florestal \\
\hline Matayba elaeagnoides Radlk. & arbusto & \\
\hline Serjania caracasana (Jacq.) Willd. & trepadeira len. & \\
\hline Serjania erecta Radlk. & arbusto & \\
\hline Serjania lethalis A.St.Hil. & trepadeira len. & \\
\hline \multicolumn{3}{|l|}{ Sapotaceae } \\
\hline Pouteria ramiflora (Mart.) Radlk. & árvore & florestal e alimentícia \\
\hline Pouteria subcaerulea Pierre ex Dubard & arbusto & \\
\hline Pouteria torta (Mart.) Radlk. & árvore & florestal e alimentícia \\
\hline Pradosia brevipes (Pierre) T.D.Penn. & arbusto & alimentícia \\
\hline \multicolumn{3}{|l|}{ Smilacaceae } \\
\hline Smilax brasiliensis Spreng. & subarbusto & medicinal \\
\hline Smilax cissoides M.Martens \& Galeotti & subarbusto & medicinal \\
\hline Smilax fluminensis Steud. & subarbusto & medicinal \\
\hline Smilax goyazana A.DC. & subarbusto & medicinal \\
\hline Smilax polyantha Griseb. & subarbusto & medicinal \\
\hline \multicolumn{3}{|l|}{ Solanaceae } \\
\hline Solanum americanum Mill. & erva & medicinal \\
\hline Solanum lycocarpum A.St.-Hil. & arbusto & medicinal \\
\hline Solanum palinacanthum Dunal & erva & \\
\hline Solanum paniculatum $\mathrm{L}$. & arbusto & medicinal \\
\hline Solanum sisymbriifolium Lam. & erva & \\
\hline \multicolumn{3}{|l|}{ Verbenaceae } \\
\hline Lantana camara L. & arbusto & med. e ornamental \\
\hline Lantana trifolia L. & arbusto & ornamental \\
\hline Lippia balansae Briq. & arbusto & ornamental \\
\hline Lippia lasiocalycina Cham. & arbusto & ornamental \\
\hline Lippia lupulina Cham. & & Ornamental \\
\hline & suarbusto c/ xilopódio & \\
\hline Lippia velutina Schauer. & arbusto & ornamental \\
\hline Stachytarpheta cayennensis (Rich.) Vahl & subarbusto & \\
\hline
\end{tabular}


Continuação...Tabela 1

\begin{tabular}{lll} 
Família/espécie & Porte & $\begin{array}{l}\text { Qualidade } \\
\text { agronômica }\end{array}$ \\
\hline Vochysiaceae & & \\
Qualea grandiflora Mart. & árvore & florestal e ornamental \\
Qualea multiflora Mart. & árvore & florestal e ornamental \\
Qualea parviflora Mart. & árvore & florestal e ornamental \\
Vochysia cinnamomea Pohl & árvore & florestal e ornamental \\
\hline
\end{tabular}

Nestas são citadas 373 espécies, distribuídas em 231 gêneros e 64 famílias. As famílias mais representadas, com 10 ou mais espécies, foram Fabaceae (58 spp.), Poaceae (24 spp.), Asteraceae (23 spp.), Euphorbiaceae (22 spp.), Myrtaceae(19 spp.), Malvaceae (18 spp.), Bignoniaceae ( 18 spp.), Apocynaceae (16 spp.), Malpighiaceae (15 spp.) e Rubiaceae (14 spp.). Essas 10 famílias contribuíram com 226 espécies, representando, aproximadamente, $60 \%$ do total; 16 famílias contribuíram com uma única espécie. Mais de 280 espécies possuem algum valor econômico (RODRIGUES; RODRIGUES, 2001; HARLEY; GIULIETTI, 2004; SILVA et al., 2001). Dois espécimes foram identificados até o nível de gênero.

Das 373 espécies identificadas, pelo menos $78 \%$ somam ervas e arbustos, aproximadamente $22 \%$ são árvores ou arvoretas e mais de $75 \%$ apresentam alguma possibilidade de uso econômico, além de representantes consideradas parentes silvestres de plantas cultivadas, dos gêneros Arachis, Passiflora e Manihot. A totalidade das espécies, desconsiderando as exóticas, está distribuída em uma única formação, Cerrado sentido restrito.

Buscou-se contemplar tanto as espécies nativas como aquelas consideradas ruderais (NETO et al., 2010), como também algumas introduzidas. A inclusão destas últimas teve por objetivo dar uma ideia dos recursos vegetais, efetivamente existente na área, para que possa servir de subsídio para solução de problemas evidentes, como a invasão de comunidades de vegetação nativas por espécies exóticas, com atenção para a presença de Melinis minutiflora que pode reduzir a regeneração arbórea e facilitar as queimadas devido ao acúmulo de matéria seca (HOFFMANN et al., 2004); e Brachiaria decumbens que também invade o Cerrado, geralmente pelas bordas e se espalham por grandes extensões (PIVELLO et al., 1999a, b) e não menos preocupante o desenvolvimento de plantas de eucalipto na área em questão. 


\section{Periódica Eletrânica

\section{CONCLUSÃO}

O número de espécies até o momento identificado, principalmente se considerarmos suas qualidades agronômicas pode ser um importante campo para coleta de sementes para experimentos em propagação, e quem sabe salvar espécies em vias de extinção. Como material taxonômico, mais de 350 espécies não deve ser insignificante; já a presença de algumas espécies exóticas é preocupante, o que nos permite sugerir o manejo adequado das mesmas. Enfim, a vegetação típica de Cerrado é uma das mais difíceis de ser reproduzida, condição que pode sustentar que qualquer fragmento deva ser conservado.

\section{REFERÊNCIAS}

AB'SABER, A.N. Os Domínios Morfoclimáticos da América do sul. Geomorfologia, Universidade de São Paulo, São Paulo, v.52, n.1, p.1-22, 1997.

APG III. An update of the Angiosperm Phylogeny Group classification for the orders and families of flowering plants: APG III. The Linnean Society of London, Botanical Journal of the Linnean Society, 161, 105-121, 2009.

DURIGAN, G.; BAITELO, J.B.; FRANCO, G.A.D.C.; SIQUEIRA, M.F. Plantas do Cerrado Paulista: imagens de uma paisagem ameaçada. São Paulo: Páginas \& Letras Editora e Gráfica, 2004. 475p.

EXÉRCITO Brasileiro 2 $2^{a}$ Companhia de Infantaria. [S.I.;s.n, 2010?] Disponível em: <http://www.exército.gov.br/060MS/2ciainf/>. Acesso em: 25 mar. 2010.

FILGUEIRAS, T.S.: BROCHADO, A.L.: NOGUEIRA, P.E.; GUALA, G.F. Caminhamento: um método expedito para levantamentos florísticos qualitativos. Caderno de Geociências, Rio de Janeiro, v.12, n.4, p.39-43, 1994.

HARLEY, R.M.; GIULIETTI, A.M. Flores nativas da Chapada Diamantina. São Carlos: RiMa, 2004. $344 p$.

HOFFMANN, W.A.; LUCATELLI, V.M.P.C.; SILVA, F.J.; AZEUEDO, I.N.C.; MARINHO, M.S.; ALBUQUERQUE, A.M.C.; LOPES, A.O.; MOREIRA, S.P. Impact of the invasive alien grass Melinis minutiflora at the savanna-forest ecotone in Brazilian Cerrado. Diversity and Distributions, Oxford, v.10, n.2, p.99-103, 2004.

MINISTÉRIO DO MEIO AMBIENTE. Parentes silvestres das espécies de plantas cultivadas. Brasília: Centro de informação e documentação Luiz Eduardo Magalhães, 2006. 42p.

MYERS, N.; MITTERMEIER, R.A.; MITTERMEIER, C.G.; FONSECA, G.A.B.; KENT, J. 2000. Biodiversity hotspots for conservation priorities. Nature, London, v.403, n. 6772, p.853-858.

NETO, M. J. et al Plantas ruderais. Campo Grande, Ed. UFMS 2010, 358 p. ISBN 978-85-7613-2998

PIVELLO, V.R.; CARVALHO, V.M.C.; LOPES, P.F.; PECCININI, A.A.; ROSSO, S. Abundance and distribution of native and invasive alien grasses in a "cerrado" (Brazilian savanna) biological reserve. Biotropica, Zurich, v.31, n.1, p.71-82, 1999a. 
PIVELLO, V.R.; SHIDA, C.N.; MEIRELLER, S.T. Alien grasses in Brazilian savannas: a threat to the biodiversity. Biodiversity and Conservation, Berlin, v.8, n.9, p.1281-1294, 1999b.

RIBEIRO, J.F.; WALTER, B.M.T. As principais fitofisionomias do bioma Cerrado. In: SANO, S.M.; ALMEIDA, S.P.; RIBEIRO, J.F. (Ed.). Cerrado: ecologia e flora. Embrapa Cerrados. Brasília, DF: Embrapa Informação Tecnológica, 2008. 2v. Cap 6, p.151-199.

RODRIGUES, V.E.G.; RODRIGUES, D.A.C. Plantas medicinais no domínio dos Cerrados. Lavras: UFLA, 2001. 180p.

SANO, E.E.; ROSA, R.; BRITO, J.L.S.; FERREIRA, L.G. Mapeamento semi-detalhado do uso da terra do Bioma Cerrado. Pesquisa Agropecuária Brasileira, Brasília, v.43, n.1, p.153-156, 2008.

SILVA, D.B.; SILVA, J.A.; JUNQUEIRA, N.T.V.; ANDRADE, L.R.M. Frutas do Cerrado. Brasília: Informação Tecnológica, 2001. 178p.

THE PLANT LIST (2010). Version 1. Published on the Internet; <http:;;www.theplantlist.org> (Acesso nos períodos de execução do trabalho). 